\title{
Von Economo's disease and postencephalitic parkinsonism responsive to carbidopa and levodopa
}

This article was published in the following Dove Press journal: Neuropsychiatric Disease and Treatment

\author{
Daniel Y Bigman \\ Bradford D Bobrin \\ Department of Psychiatry, Cooper \\ University Hospital, Cooper \\ Medical School of Rowan University, \\ Camden, NJ, USA
}

\begin{abstract}
Postencephalitic parkinsonism (PEP) is currently perceived as having a very close etiologic relationship with encephalitis lethargica (von Economo's disease [EL]), with PEP developing immediately after the acute phase of EL or at some time later. EL was classically described by von Economo and has somnolent-ophthalmoplegic, hyperkinetic, and amyostaticakinetic forms. Previous cases have reported success with levodopa in PEP. We report a case with PEP showing success after administration of levodopa and carbidopa. This case would support the hypothesis that this syndrome effects dopamine neurotransmission.

Keywords: von Economo, encephalitis lethargica, postencephalitic parkinsonism, carbidopa, levodopa, catatonia
\end{abstract}

\section{Introduction}

Encephalitis lethargica (von Economo's disease, sleeping disease; EL) occurred in epidemic proportions between 1916 and 1929. The clinical manifestations were described in detail by the Austrian physician von Economo who described the atypical encephalitis that predominantly affected the basal ganglia resulting in movement, psychiatric, and sleep disorders. ${ }^{4}$ Parkinsonism was the classic movement disorder, but hyperkinetic movements were often concurrent. ${ }^{2}$ Psychiatric disorders were common, and EL has shown similarities with catatonia. ${ }^{4}$ There have been a number of contemporary reports of EL in the recent decades and diagnostic criteria have been proposed. ${ }^{2,3}$ Contemporary postencephalitic parkinsonism (PEP) is related to a postencephalitic state in which there is subsequent degeneration of the basal ganglia leading to clinical parkinsonism. ${ }^{4}$ While the etiology remains uncertain, modern reviews have EL associated with PEP due to post-infectious and/or autoimmune phenomenology. ${ }^{1}$ We report a case that showed movement disturbance, psychiatric, and sleep disturbance. In addition, we describe the improvement of symptoms once levodopa and carbidopa are initiated, recurrence of symptoms when withheld, and resolution of symptoms with re-administration of levodopa and carbidopa.

\section{Case} Department of Psychiatry, Cooper University Hospital, Cooper Medical School of Rowan University, 40I Haddon Avenue, Suite 352, Camden, NJ 08103, USA

Tel +l 8567577853

Email bigman-daniel@cooperhealth.edu
A 66-year-old Caucasian male was admitted to the hospital for altered mental status. The patient has been a resident at a psychiatric facility for bipolar disorder.

The patient's history was obtained by a Geriatric Psychiatrist at the Psychiatric facility who has been following the patient, Baseline mental state and function is reported as ambulatory. The patient interacts with fellow residents and speaks 
appropriately with linear process. The patient is not noted to have any tremors or movement disturbances. Gait is recalled as steady. According to the reports, the patient was on olanzapine $5 \mathrm{mg}$ nightly for a few months, which was discontinued due to drowsiness 8 weeks prior to admission. The patient has remained on standing valproic acid extended release tablet $1,000 \mathrm{mg}$ at bedtime and clonazepam $0.25 \mathrm{mg}$ PO BID for anxiety and irritability.

Five days prior to admission, the patient was noted to be febrile; the patient remained bedridden with continuous sleep, fixed stare and movement similar to oculogyric crisis, and intermittent rigidity. Valproic acid was held and the patient was started on lorazepam q8h for 3 days for suspected catatonic features. However, the patient's condition worsened. The patient was then recommended escalation of care and presented to the hospital and admitted to the medical service.

Once admitted, the patient was medically worked up by the critical care team and found to have a blood pressure of $100 \mathrm{~s} / 70 \mathrm{~s}$, heart rate of $110 \mathrm{~s}$, and oxygen saturation of $99 \%-100 \%$ on $2 \mathrm{~L}$ nasal cannula. The patient remained unresponsive rigid presentation, and disoriented. Intravenous cefepime, vancomycin, and metronidazole were initiated. Head CT without contrast reported no bleed or acute findings and noted incidental arachnoid cyst.

Psychiatrist was consulted to evaluate for altered mental status with concern for catatonic features and to rule out neuroleptic malignant syndrome or serotonin syndrome. On examination, the patient appeared ill, with no obvious lesions, head without any tenderness or deformities, pupils equally round and reactive to light and accommodation, fixed stare, poor dentition, no oropharyngeal erythema or injection, S1 S2 audible without murmur, chest clear to auscultation without wheeze rhonchi or rales, abdomen soft non-tender and non-distended, palpable pulses throughout without any edema. The patient appeared alert, responded to voice, with incomprehensible sounds, moves all extremities spontaneously, visible mild resting tremor to distal extremities, with reflexes intact, and found to be hypertonic throughout all 4 extremities. Due to the patient's fever, autonomic instability, rigid appearance, and chronic psychiatric disorder, acute catatonia was suspected with etiology uncertain. The patient was recommended to start lorazepam $2 \mathrm{mg}$ IV every 8 hours.

By day 3, the patient's condition remained the same with minimal improvement in bradykinesia, rigidity, and fixed stare. A resting tremor and decreased glabellar reflex were noted. The patient's home dose of valproic acid was reinitiated. The neurology team was consulted for further evaluation of mental status changes and the team recommended a lumbar puncture and MRI of the brain. Due to suspicion of dental infection, the oral and maxillofacial team was consulted and excision of the carious non-restorable teeth was performed on day 4. Operative report mentions no obvious abscess, fluid collection, or gross infection. The patient tolerated anesthesia, and the perioperative period was without notable oral or maxillofacial complications. The patient's presentation on days 5-7 remained the same and lorazepam was titrated down and discontinued. In addition, the patient failed a swallow study and a nasogastric tube was placed for nutritional tube feeds.

On day 7, an EEG was performed, which reported "an abnormal EEG due to generalized background slowing. Generalized background slowing may be due to toxic/metabolic/ infectious state, or alternatively due to medication effect. No seizures recorded. Clinical correlation is recommended." Blood cultures, urine culture, and preliminary lumbar puncture cultures reported no growth. Broad-spectrum antibiotics were discontinued. A trial of carbidopa and levodopa 25/100 mg every 8 hours was initiated. A review of comprehensive laboratory and diagnostic testing is resulted in Box 1. Axial and sagittal plane of brain MRI is displayed in Figure 1.

On days $8-10$, the primary team members and psychiatry team were in agreement of clinical improvement; the patient was able to wiggle toes on command, with less rigidity and cessation of tremor, and able to track eyes with personnel. On day 10 , the carbidopa and levodopa dose was increased to $25 / 250 \mathrm{mg}$ every 8 hours.

On day 14 , family members reported dramatic improvement in mental status with recognition of family members, relying to name, responding with one-word answers. Lower extremities were no longer rigid. Upper extremities were with some resistance. Speech pathology noted improvement in swallow function and recommended gentle diet initiation.

On day 16, a comprehensive discussion was done with the primary medical, neurology, and psychiatry teams with the family; due to the patient's atypical presentation and potential activating properties of carbidopa and levodopa, a trial to discontinue carbidopa and levodopa was entertained to truly consider a postencephalitic parkinsonian state. This was agreed with all clinicians and family members were in agreement.

During days 16-21, the patient was weaned off the carbidopa and levodopa initially by reduction in dose from $25 / 250$ to $25 / 100 \mathrm{mg}$ every $8-12$ hours and then discontinued. The patient's appearance worsened with notable recurrence of fixed stare, mutism, and rigidity. 
Box I Laboratory results and diagnostics

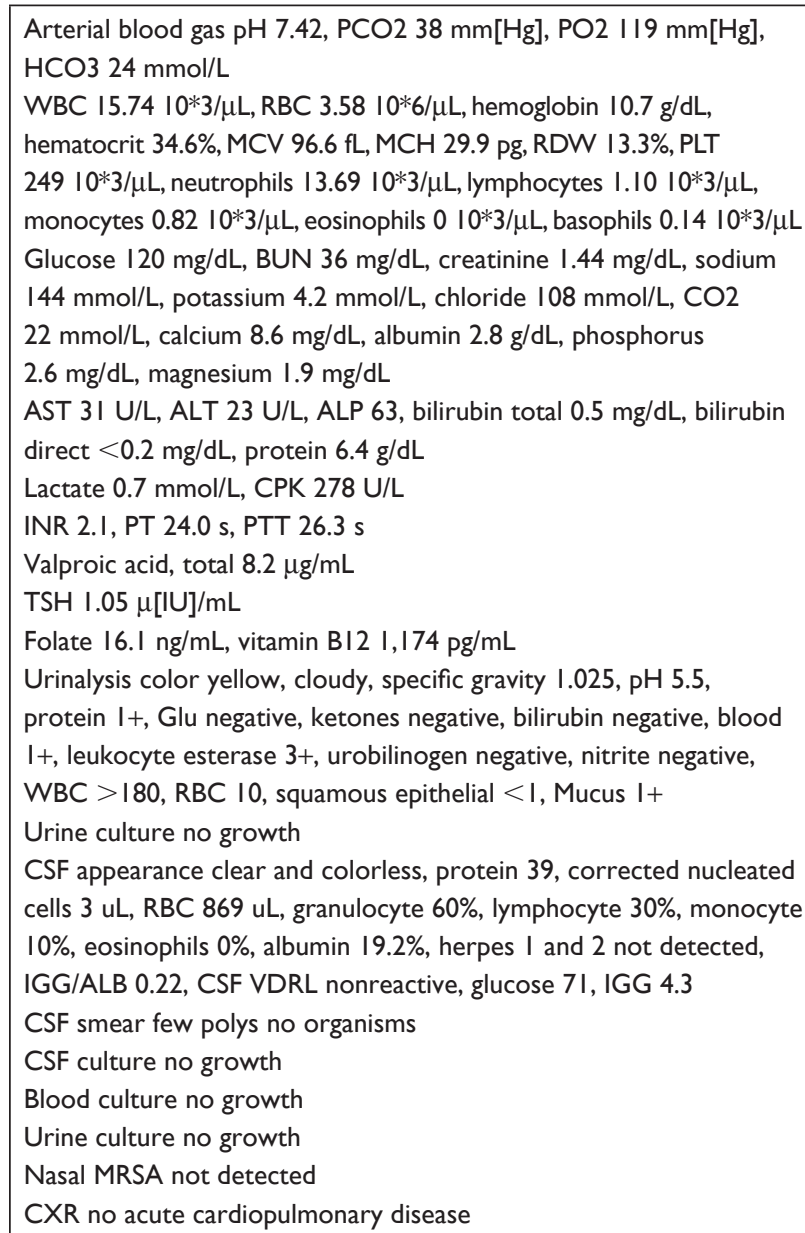

Abbreviations: WBC, white blood cell; RBC, red blood cell; MCV, mean corpuscular volume; $\mathrm{MCH}$, mean corpuscular hemoglobin; RDW, red blood cell distribution width; PLT, platelet; BUN, blood urea nitrogen; AST, aspartate aminotransferase; ALT, alanine aminotransferase; ALP, alkaline phosphatase; CPK, creatine phosphokinase; INR, international normalized ratio; PT, prothrombin time; PTT, partial thromboplastin time; TSH, thyroid stimulating hormone; CSF, cerebral spinal fluid; IGG/ALB, immunoglobulin G / albumen; CSF VDRL, cerebral spinal fluid venereal disease research laboratory test; MRSA, methicillin resistant staph aureus; CXR, chest x-ray (radiograph).
On day 21 , the patient failed speech therapy's swallow trail and it was recommended that they have nothing by mouth. Due to these worsening clinical features and concern for swallow and airway compromise, carbidopa and levodopa were re-initiated.

On day 23, the patient had a dramatic improvement to clinical presentation; resolution of rigidity, tracking eyes, increased words when conversing, and sleep cycle were restored. The patient was oriented to self, place, and year. No tremor to the extremities or obvious bradykinesia was noted. The patient started to ambulate out of bed with assistance. In addition, the patient passed the speech therapy swallow study, began to tolerate meals, and selffed with meal trays. The patient was discharged on day 25 and was transferred back to a rehabilitation and skilled nursing facility.

Ninety-day follow up is notable for continued administration of carbidopa and levodopa 25/200 mg controlled release tablet orally twice daily. The patient and family report that the patient is back to baseline function and residing at a long-term care psychiatric facility.

\section{Ethics}

The authors obtained written informed consent from the patient to have case details and accompanying images published.

\section{Discussion}

The case reported identifies a patient with a chronic psychiatric disorder and new onset persistent fever without an obvious source, and presents with unretractable catatonic
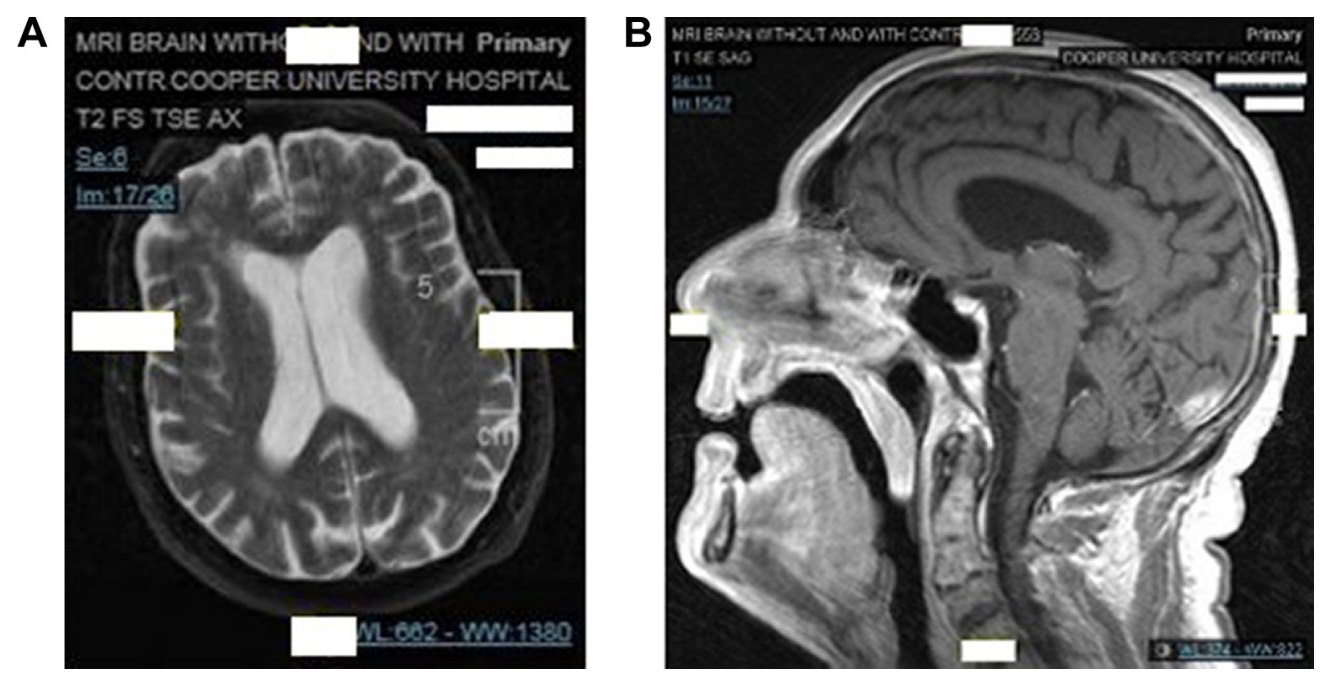

Figure I MRI of the brain with contrast.

Notes: (A) T2WI axial view. (B) TIWI sagittal view. Both views demonstrate nonspecific dural enhancement.

Abbreviations: MRI, magnetic resonance imaging; TIWI, TI-weighted image; T2WI, T2-weighted image. 
features, which evolved to a parkinsonism-like presentation that clinically responded to carbidopa and levodopa administration. Catatonia is marked by heterogeneous signs that are observed or elicited; most common are immobility, stupor, negativism, staring, and echolalia. ${ }^{5,6}$ The subtypes are based on the specific nature of movement disturbance and other associated features: retarded - mutism, inhibited movement, posturing, rigidity, negativism, and starting; malignant - fever, autonomic instability, delirium, and rigidity; excited - excessive and purposeless motor activity, restlessness, stereotypy, impulsivity, frenzy, agitation, and combativeness. ${ }^{5}$ Benzodiazepines and then electroconvulsive therapy (ECT) are considered as the first line treatment of choice in suspected catatonia. ${ }^{6}$ In cases with poor response, there have been reports describing other treatments such as valproate, ${ }^{7}$ zolpidem, ${ }^{8}$ memantine, ${ }^{9}$ and bromocriptine. ${ }^{10}$

While this patient presented with features similar to malignant catatonia, the patient failed a trial of lorazepam prior to hospital admission as well as a 5-day trial once admitted. This brought into question of resolving any underlying illness or alternate etiologies, especially, since $60 \%-80 \%$ of reported catatonia cases respond to full remission with benzodiazepine or ECT. ${ }^{5}$ Although ECT was considered by the psychiatry team, the patient's presentation of distal extremity tremors, axial dystonia, dysphagia, decreased spontaneous eye blink rate, and speech impairment suggested highly atypical and unreported symptoms of catatonia, which warranted revisiting the differential diagnoses. A literature search of valproic acid-induced parkinsonism will yield multiple case reports, series, and reviews indicating a likely adverse effect. Valproic acid-induced parkinsonism has been reported after months of therapeutic administration and is generally reversible with the withdrawal of valproate. ${ }^{11} \mathrm{We}$ entertained the idea of valproic acid-induced parkinsonism. However, the patient is reported to have been chronically administered a sub-therapeutic dosage of valproate for a couple of years. In addition, 3 days prior the admission, the nursing facility held valproic acid, which was not re-started until day 3-4 of hospital admission. During the period of withholding this patient's valproic acid, there was no clinical improvement of symptoms and some subjective argument for worsened appearance.

Oliver Sacks' descriptions of L-dopa-induced “Awakening" of PEP patients described a similar experience with dyskinesias necessitating cessation of L-dopa. ${ }^{12}$ This phenomenology is based on the hypothesis that a combination of dopamine depletion followed by hypersensitive dopamine receptors is occurring in EL, resulting in a tenuous dopamine neurotransmission imbalance. ${ }^{2}$ Behavioral disorders are also characteristic features of EL, and are almost universal in historical and contemporary cases (agitation, catatonia, depression, apathy). ${ }^{1}$ Hallmark symptoms of EL generally suggest extrapyramidal, behavioral, and sleep disturbances. ${ }^{13}$ Historical EL describes mainly parkinsonism, dystonia, tongue and jaw tremor, abnormal postures, and truncal instability, ${ }^{1}$ much of which this case presented.

The pathophysiology of EL is not fully understood. Although commonly occurring in the post-infectious setting, there is no consistent infectious precipitant nor evidence of microorganisms invading the central nervous system. ${ }^{2}$ This case presented with a potential toxic and/or metabolic encephalopathy with postencephalitic parkinsonism. Some case reports have been successful with the use of lorazepam for agitation in suspected EL. ${ }^{2}$ Alternatively, electro-convulsive therapy has been successfully used in catatonic EL. ${ }^{14}$ Another case report noted success with steroids in suspected EL, which would support the inflammatory hypothesis of concentrated inflammatory infiltrates to the midbrain and basal ganglia. ${ }^{15}$

Recent discoveries appear to point towards a variety of different etiologies of EL-like disorders and within the context of autoimmune encephalitis. There have been increasing descriptions of novel anti-neuronal and antiglial antibodies that are associated with paraneoplastic and non-paraneoplastic neurological syndromes. ${ }^{16}$ Autoimmune encephalitis and paraneoplastic encephalitis were entertained since EL and PEP have been suggested to be autoimmune encephalitides. CSF autoimmune antibodies or IVIG/plasma exchange was not obtained or performed due to the improvement of symptomatology. In addition, the initial CSF analysis did not yield elevated protein and brain MRI did not report abnormal signals, which would be atypical for autoimmune encephalitis.

The clinical presentation and investigative findings to the case presented confirm the role of dopamine in suspected EL and PEP. While the patient may have initially presented with catatonic features, the patient poorly responded to lorazepam and dramatically improved with a dopamine agonist. The etiology of this case presentation remains unclear. While we concurred to a diagnosis of PEP, there was uncertainty in confirming the suspected EL. However, there appeared to be an encephalitic process with new onset parkinsonism, which dramatically improved with the administration of carbidopa and levodopa. While historical contexts appear to still have blurred lines in the definitions of von Economo's 
encephalitis, contemporary EL, or PEP, this case supports the considerable reports that suggest the role of dopamine agonists in suspected dopamine-deleted states. Further study should be considered.

\section{Acknowledgment}

We would like to thank Jia Z Cheng, MD, Department of Neurology, Cooper Neurological Institute, Cooper University Hospital, Cooper Medical School of Rowan University, Camden, NJ, USA, for assistance with manuscript editing.

\section{Author contributions}

Both authors managed the patient, contributed toward data analysis, drafting and critically revising the paper, gave final approval of the version to be published, and agree to be accountable for all aspects of the work.

\section{Disclosure}

The authors report no conflicts of interest in this work.

\section{References}

1. Vilensky JA, Gilman S, McCall S. Does the historical literature on encephalitis lethargica support a simple (direct) relationship with postencephalitic Parkinsonism? Mov Disord. 2010;25(9):1124-1130.

2. Dale RC, Webster R, Gill D. Contemporary encephalitis lethargica presenting with agitated catatonia, stereotypy, and dystonia-parkinsonism. Mov Disord. 2007;22(15):2281-2284.
3. Kim R, Shin CW, Kim HJ, Jeon BS. Postencephalitic parkinsonism responsive to a dopamine agonist: a case report. Parkinsonism Relat Disord. 2015;21(6):667-668.

4. Von Economo C. Encephalitis Lethargica: Its Sequelae and Treatment. Newman KO, translator. London: Oxford University Press; 1931.

5. Mann SC, Caroff SN, Campbell EC, et al. Malignant catatonia. In: Frucht SI, Fahn S, editors. Current Clinical Neurology: Movement Disorder Emergencies: Diagnosis and Treatment. Totowa, NJ: Humana Press; 2005:53.

6. Hawkins JM, Archer KJ, Strakowski SM, Keck PE. Somatic treatment of catatonia. Int J Psychiatry Med. 1995;25(4):345-369.

7. Krüger $S$, Bräunig P. Intravenous valproic acid in the treatment of severe catatonia. J Neuropsychiatry Clin Neurosci. 2001;13(2):303-304.

8. Thomas P, Rascle C, Mastain B, Maron M, Vaiva G. Test for catatonia with zolpidem. Lancet. 1997;349(9053):702.

9. Carpenter SS, Hatchett AD, Fuller MA. Catatonic schizophrenia and the use of memantine. Ann Pharmacother. 2006;40(2):344-346.

10. Tang CP, Leung CM, Ungvari GS, Leung WK. The syndrome of lethal catatonia. Singapore Med J. 1995;36(4):400-402.

11. Mahmoud F, Tampi RR. Valproic acid-induced parkinsonism in the elderly: a comprehensive review of the literature. Am J Geriatr Pharmacother. 2011;9(6):405-412.

12. Sacks OW, Kohl M. L-dopa and oculogyric crises. Lancet. 1970; 2(7665):215-216.

13. Howard RS, Lees AJ. Encephalitis lethargica. A report of four recent cases. Brain. 1987;110(Pt 1):19-33.

14. Cheyette SR, Cummings JL. Encephalitis lethargica: lessons for contemporary neuropsychiatry. J Neuropsychiatry Clin Neurosci. 1995;7(2): $125-134$.

15. Blunt SB, Lane RJ, Turjanski N, Perkin GD. Clinical features and management of two cases of encephalitis lethargica. Mov Disord. 1997; 12(3):354-359.

16. Finn ES. Autoimmune Encephalitis, History and Current Knowledge. Copenhagen: Statens Serum Institute; 2013.
Neuropsychiatric Disease and Treatment

\section{Publish your work in this journal}

Neuropsychiatric Disease and Treatment is an international, peerreviewed journal of clinical therapeutics and pharmacology focusing on concise rapid reporting of clinical or pre-clinical studies on a range of neuropsychiatric and neurological disorders. This journal is indexed on PubMed Central, the 'PsycINFO' database and CAS,

\section{Dovepress}

and is the official journal of The International Neuropsychiatric Association (INA). The manuscript management system is completely online and includes a very quick and fair peer-review system, which is all easy to use. Visit http://www.dovepress.com/testimonials.php to read real quotes from published authors. 